\title{
Die sprachliche Situation in der alten deutschen Sprachinsel Kostenthal/Gościęcin im Unterschied zu anderen Teilen Oberschlesiens
}

\begin{abstract}
Odmiany języka niemieckiego występujące poza zwartym obszarem niemieckojęzycznym tworzą wskutek interakcji z koegzystującymi w tych regionach językami swoisty rodzaj wariantów kontaktu. Nie oznacza to bynajmniej, że odmiany te mają charakter homogeniczny. Niniejszy artykuł unaocznia na przykładzie wariantu gościęcińskiego, że niemczyzna górnośląskich wysp językowych odbiega od języka niemieckiego występującego na większości terenów Górnego Śląska, przy czym różnice te wynikają z innego historycznego rozwoju tychże wariantów. Nadto artykuł ma być próbą krótkiego nakreślenia stanu dotychczasowych badań językoznawczych dotyczących Śląska i wskazać na niektóre istniejące jeszcze luki badawcze.
\end{abstract}

Varietäten des Deutschen außerhalb des zusammenhängenden deutschen Sprachgebiets stellen infolge der Interaktion mit den Umgebungssprachen eine Art Kontaktvarietäten dar. Dies bedeutet jedoch nicht, dass es sich dabei um homogene Erscheinungsformen des Deutschen handelt. Anhand des Sprachinseldeutschen von Kostenthal wird im vorliegenden Beitrag veranschaulicht, dass sich die oberschlesischen Sprachinseln vom Deutschen im Großteil Oberschlesiens nachweislich unterscheiden, wobei diese Differenzen auf die unterschiedliche Entwicklungsgeschichte dieser Varietäten zurückführbar sind. Ferner wird der Versuch unternommen, einen Überblick über den Stand der linguistischen Untersuchungen zu Schlesien zu schaffen und einige noch bestehende Forschungslücken aufzuzeigen.

The varieties of German spoken beyond the closed German speaking territories are, as a result of the interaction with the surrounding languages, a kind of contact varieties. Still, it doesn't mean that those vernaculars are homogenous. The present paper shows on the basis of the vernacular of Gościęcin that the varieties of the Upper Silesian speech islands vary from the German which is spoken in the most parts of Upper Silesia. The differences result from different historical developments of the varieties. 
Felicja Księżyk

The aim is also to outline the state of linguistic research on Silesia and to point some still existing research gaps.

Das Gebiet Schlesiens stellt seit jeher eine besondere, da multilinguale Region dar. In einer Sprachenzyklopädie vom Anfang des 19. Jhd.s liest man dazu Folgendes:

In Ober-Schlesien ließen sich weniger Deutsche nieder [...]. In der letzten Hälfte des fünfzehnten Jahrhunderts breiteten sich die Hussiten in Ober-Schlesien aus, und nun verdrängte die Böhmische Sprache die Deutsche und Lateinische aus den Urkunden und Gerichtshöfen, besonders in den Fürstenthümern Oppeln und Ratibor. Beyde wurden oft verpfändet, und besonders 1645-1666 an Polen, wodurch sich das Polnische wieder hob. Die in Ober-Schlesien üblichen Sprachen beweisen die Vermischung der Völker. In Troppau und Jägerndorf spricht man Deutsch, bis auf einige Gegenden, wo mit Polnisch vermischtes Mährisch herrscht; in Oppeln und Ratibor aber ein verdorbenes Polnisch, welches ein Pole nur schwer verstehet. Man nimmt Deutsche Wörter und gibt ihnen Polnische Endungen und Wortfügungen. (ADELUNG 1809:669f.)

Aufgrund der sprachlichen Vielfalt und der gegenseitigen Einflüsse der in Schlesien herrschenden Sprachvarietäten erscheint es zutreffend, in Anlehnung an FÖLDES (2002:79) von einer Region von „Kontaktvarietäten“ zu sprechen. Zwar setzt das sprachwissenschaftliche Interesse an Schlesien, so WIKTOROWICZ (2001:267), schon im 19. Jhd. ein (berücksichtigt man die z. T. bereits im 18. Jhd. erschienenen Arbeiten, etwa die Schlesischen Idiotika, die ADELUNG in dem zweiten Mithridates ${ }^{1}$ anführt, so dürfte man sogar von früheren Ansätzen der Beschäftigung mit Schlesien sprechen), doch lange Zeit wurde der in dem obigen Zitat angesprochene mehrsprachige Charakter Schlesiens, ,der gegenseitige Einfluss der deutschen und polnischen Mundart aufeinander [...] völlig aus der wissenschaftlichen Analyse verdrängt; stattdessen konzentrierte man sich - insbesondere in der Nazizeit [...] auf die so genannte ,deutsche Kulturarbeit“ in Schlesien“ (WIKTOROWICZ 2001:269). Zudem sind die im Laufe der Zeit erbrachten Untersuchungsergebnisse nicht jederzeit gleichermaßen zuverlässig. Während die Wissenschaftler in der ersten Phase der Auseinandersetzung mit den Sprachbeziehungen (Wiktorowicz setzt sie bis zum Ende des Ersten Weltkriegs an) um Objektivität und Exaktheit bemüht

1 Siehe dazu Adelung (1809:217). Bei dem Werk handelt es sich um eine Art Sprachenzyklopädie, die auf die Erkundung der Herkunft und Verwandtschaft der Sprachen zielt. Der Name führt auf den polyglotten König von Pontus zurück, der im 1. Jhd. v. Chr. lebte und unterschiedlichen Geschichtsquellen zufolge zwischen 22 und 50 Sprachen beherrschte. Vgl. TRABAnt, Jürgen: Mithridates in Berlin: http://www.berliner-klassik.de/projekte/publikationen/online/trabant (30.07.2007). 
sind, dominiert in der zweiten Beschäftigungsphase (seit dem Ende des Ersten Weltkriegs) eine tendenziöse, nationalistisch-chauvinistische Sichtweise. Ebenfalls vielfach ideologisch geprägt ist die wissenschaftliche Beschäftigung in der dritten Phase (nach dem Zweiten Weltkrieg) - die entstehenden Arbeiten sollten das Urpolnische der ehemaligen deutschen Gebiete beweisen und somit deren Zugehörigkeit zu Polen legitimieren. Mit dem Nachlassen des politischen Drucks von außen in den 70er Jahren lässt sich die letzte Phase der Beschäftigung mit den sprachlichen Verhältnissen in Schlesien ansetzen, in der (insbesondere in den 90er Jahren) erneut objektive Analysen vorgelegt wurden (vgl. WIKTOROWICZ 2001:265-272).

Die Beschäftigung mit Kostenthal, einer sekundären deutschen Sprachinsel ${ }^{2}$, gelegen in Oberschlesien zwischen Kandrzin-Cosel/Kędzierzyn-Koźle und Oberglogau/Głogówek, setzt in der zweiten Phase ein, als deutsche Forscher die Geschichte der deutschen Siedlungstätigkeit als ein interessantes Untersuchungsobjekt entdeckten. ${ }^{3}$ Die aus dieser Zeit stammenden Veröffentlichungen zu Kostenthal liefern in erster Linie historische und volkskundliche Auskünfte. Die umfassendsten Informationen über das Sprachinseldorf (zu Geologie, Siedlungsgeschichte, Volkskunde, Demographie und Sprache) finden sich in einem Sonderheft des „Oberschlesiers“, der Nachlese eines Lehrgangs, „,der (1936) in Kostenthal [...] stattfand und [...] seinen Teilnehmern einen Einblick [...] in das volkskundliche Leben [...] [des] Dorfes" (SCHERHOLZ 1937:543) gewähren sollte. Der Wahl Kostenthals als Schulungsort lag die Absicht zugrunde, ,der immer mehr geschwundenen Kostenthaler Mundart nachzugehen" (SCHERHOLZ 1937:544) und durch die Veröffentlichungen den Dorfbewohnern einsichtig zu machen, dass die kostenthalerische Mundart ein kostbares Gut ist, die es vor dem Aussterben zu bewahren galt (SCHERHOLZ 1937:545). Denn anders als im überwiegenden Teil Oberschlesiens, wo zu Beginn des 16. Jhd.s die deutschsprachigen Siedlungen polonisiert worden waren (MORCINIEC 1999:293 und KNEIP 2000:23), konnte sich der im 13. Jhd. von den Siedlern mitgebrachte deutsche Dialekt in Kostenthal jahrhundertelang aufrechterhalten, weshalb der Ortschaft der Status einer Sprachinsel zugesprochen wurde. Während nämlich das Deutsche im Großteil Oberschlesiens

2 Sekundären Charakters ist die Sprachinsel deswegen, weil deren Siedler nicht unmittelbar aus Deutschland kamen, sondern aus älteren deutschen Sprachinseln (KUHN 1934:45). Zum Sprachinselkonzept siehe MATTHEIER (2003:16).

3 WikTOROWICZ (2001:268) beanstandet allerdings an diesen Untersuchungen die Tatsache, dass der „Anteil der deutschen Siedler [...] von den deutschen Autoren häufig zu hoch veranschlagt [wurde]“. 
Felicja Księżyk

aus dialektologischer Sicht für nicht bodenständig erachtet wird ${ }^{4}$, weist Kostenthal dialektale Hintergründe auf.

Ähnlich wie bei anderen Sprachinseln suchte man auch in den in der ersten Hälfte des 20. Jhd.s veröffentlichten Artikeln über Kostenthal vordergründig der Frage nach der Herkunft der Siedler nachzugehen (s. dazu WEINELT 1938 und 1939, sowie SCHWARZ 1935:205-211). Die eindringlichste Beschreibung der kostenthalerischen Lautlehre lieferte in dieser Zeit WEINELT (1938) mit seinem Artikel Sprache und Siedlung der oberschlesischen Sprachinsel Kostenthal. Kürzere Beiträge zu der Kostenthaler Varietät des Deutschen, die lautlichen Verhältnisse und Affinitäten zu der Umgegend betreffend, verfasste FRIEMEL (1937, 1937a und 1938). Einige Mundartproben aus dem Sprachinseldorf veröffentlichten überdies GRAEBISCH (1929) und MAK (1929).

Die Verschiedenheit der Kostenthaler Erscheinungsform des Deutschen vom Deutschen im überwiegenden Teil Oberschlesiens findet ihren Niederschlag darin, dass bei den Untersuchungen des oberschlesischen Deutsch andere Forschungsziele verfolgt werden. Berühren die vorkriegszeitlichen Veröffentlichungen das oberschlesische Deutsch nur am Rande (PELKA 2006:32), so scheinen die späteren Untersuchungen vor allem dem Zweck verpflichtet, den Einfluss des Polnischen bzw. der polnischen schlesischen Dialekte auf das oberschlesische Deutsche herauszustellen (s. OLESCH 1970 und 1987, REITER 1960, Rohfleisch 2001, PelKa 2006). Zwar beteuert PelKa (2006:11), dass „nicht alle Eigentümlichkeiten der in Oberschlesien gesprochenen deutschen

4 Es handelt sich dabei nämlich nicht um eine Fortsetzung des mittelalterlichen Sprachzustandes, sondern eine im schulischen Unterricht erworbene, von Sprachkontaktprozessen betroffene, mehr oder weniger standardnahe Umgangssprache. Durch den Sprachkontakt mit den polnisch-schlesischen Dialekten, eine Art Bildungsbilingualismus, entwickelte sich das im schulischen Unterricht erworbene Deutsch allmählich zu einer Kontaktvarietät. Siehe dazu BELLMANN (1971:10, 16), ROHFLEISCH (2001:165f.) und REITER (1960:64). Damit korrespondiert auch die Beschreibung der Sprach- und Varietätenverteilung bei der deutschsprachigen Minderheit in Oberschlesien in RIEHL (2004:161). Danach ist die Erstsprache der vor 1932 geborenen Generation das Standarddeutsche (und nicht wie bei anderen deutschsprachigen Minderheiten in Mittel- und Osteuropa ein deutscher Dialekt), ihre Zweitsprache ist hingegen das Polnische. Bei der zwischen 1932 und 1952 geborenen Generation fungieren beide Sprachen als Erstsprachen, die zwischen 1952 und 1975 Geborenen verfügen lediglich über Polnischkenntnisse. Die letzte, nach 1975 zur Welt gekommene Generation weist gegenüber der ältesten einen Gebrauchswandel auf: Ihre Erstsprache ist das Polnische, die Zweitsprache ist das Deutsche. 
Sprache durch einen Einfluss des Standardpolnischen und seiner Varietäten erklärt werden [können]", und sie lehnt zu Recht die zuweilen vertretene These ab, ,das Deutsch der Oberschlesier sei ein Schriftdeutsch mit polnisch-mundartlichen Einflüssen und stehe auf dem phonologischen System der schlesischen Mundart $[\ldots]$ als Simplifizierung der realen Gegebenheiten $[\ldots]$ “ (PELKA 2006:230), zugleich gesteht sie allerdings dieser Beeinflussung eine besondere Rolle zu, indem sie feststellt, dass gerade der ständige Kontakt des Deutschen mit der polnischen oberschlesischen Mundart bzw. der polnischen Standardsprache konstitutiv für die Herausbildung der spezifischen, vom Standarddeutschen zu unterscheidenden Eigenarten des oberschlesischen Deutsch ist (PELKA 2006:11). Im Gegensatz dazu erscheint es im Falle des Kostenthaler Deutsch aufgrund seiner dialektalen Hintergründe nicht mehr so naheliegend, die Ursachen für manche vom Standard abweichenden Erscheinungen innerhalb der deutsch-polnischen Systemkontraste zu suchen, da darin vielfach auch eine Bewahrung des historischen Sprachbestandes gesehen werden kann.

Angesichts des Fehlens einer umfangreicheren Arbeit zur kostenthalerischen Varietät des Deutschen wurde im Rahmen eines Dissertationsprojekts ${ }^{5}$ versucht, diese Forschungslücke zu schließen. Die Untersuchung verfolgte mehrere Teilziele: Erstellung einer Dokumentation des Kostenthalerischen und Prüfung, inwiefern das rezente Deutsch in Kostenthal noch dialektal geprägt ist und ob folglich noch von einer mittelhochdeutschen Kontinuität dieser Erscheinungsform des Deutschen die Rede sein kann. Die Untersuchungsgrundlage für die phonologische und morphologische Analyse bildeten Daten, die mit Hilfe eines Fragenkatalogs erhoben wurden. Zugängliches sprachliches Material lieferten außerdem Tonaufnahmen von Dialektsprechern aus den ehemaligen deutschen Ostgebieten (,Flüchtlingen oder Vertriebenen'), die im Deutschen Spracharchiv im Institut für Deutsche Sprache, Mannheim, archiviert sind. ${ }^{6}$ Unter den Kennnummern IV/232, IV/233 und IV/234 liegen dort drei Aufzeichnungen der Kostenthaler Mundart aus dem Jahre 1962 vor. Der Dialektalitätsgrad des rezenten Kostenthaler Deutschen (die sog. ,Systemkontrast-Dialektalität ${ }^{67}-$ d.h. der Grad der Abweichung von der Standardsprache)

5 KsIĘŻYK, Felicja: Die deutsche Sprachinsel Kostenthal - Geschichte und Gegenwart. Opole. Die Dissertation wurde 2005 verteidigt und soll demnächst im trafoVerlag erscheinen.

6 Zu den Beständen des Deutschen Spracharchivs vgl. http://www.ids-mannheim.de/ DSAv.

7 Siehe dazu HERRGEN / SCHMIDT (1985), die die Unterscheidung zwischen ,Systemkontrast-“ und ,Hörerurteildialektalität' einführten. 
wurde dagegen anhand von Aufnahmen freier Rede ermittelt. In die Untersuchung einbezogen wurden lediglich Vertreter der ältesten Generation, da teilweise schon Mittsechziger behaupten, dass ihnen die von den Eltern oder Nachbarn gesprochene Mundart unverständlich sei. Ein Vergleich des Spontanmaterials und der Fragebuchaufnahmen zeigt zudem, dass die heute von den Gewährspersonen gesprochene Erscheinungsform des Deutschen historisch gesehen überwiegend eine differente Stufe der Sprachentwicklung als die Fragebuchaufnahmen darstellt. Die Träger des heutigen Kostenthalerischen selbst (Vertreter der kleinen Gruppe, die im familiären Bereich am Gebrauch des Deutschen festhalten) beteuern, sie sprächen nicht mehr den Kostenthaler Dialekt, sondern ,Hochdeutsch'. Diese Behauptung erhärtete sich durch die quantitative Analyse ${ }^{8}$, aus der hervorging, dass sich der Kostenthaler Substandard zumindest auf der phonetischen Ebene im oberen Bereich der DialektStandard-Achse platziert - der ermittelte durchschnittliche Dialektalitätswert übertrifft nämlich nur geringfügig denjenigen der Sprache von Gemeinderatsmitgliedern bei Gemeinderatssitzungen. ${ }^{9}$

Bei der qualitativen Analyse der freien Rede konnte allerdings oftmals die mhd. Kontinuität der in Kostenthal gesprochenen deutschen Varietät verfolgt werden. Es war möglich, remanente Dialektmerkmale herauszuarbeiten, die die Sprecher auch bei deklarierter Benutzung der Standardsprache beibehalten. Diese Standarddivergenz tritt in allen Subsystemen der Sprache zutage. Im Folgenden sollen einige Beispiele angeführt werden, die die Dialektalität des rezenten Kostenthaler Deutsch nachvollziehbar illustrieren können.

So fällt im Bereich der Phonetik auf, dass bei vorausgehendem /r/ die s-Palatalisierung beibehalten wird, etwa in erscht, andersch. Allerdings handelt es sich bei dieser stellungsbedingten Wiedergabe um keine ortsexklusive Realisierung, EBERTH (1846:221-226) zählt diese Erscheinung neben einigen anderen Abweichungen von der Schriftsprache, von denen manche ebenfalls Beispiele

8 Dabei wurde auf das 1989 von Herrgen und Schmidt vorgeschlagene und seitdem bereits mehrfach angewandte Messverfahren zurückgegriffen, das in HERRGEN / LAMELI (2001) ausführlich dargelegt wird und sich ausschließlich als ein phonetisches Messinstrument versteht.

9 In einer vergleichenden diachronen Untersuchung der Aufnahmen von Gemeinderatssitzungen in den Städten Mainz und Neumünster ermittelte LAMELI (2003:8f.) folgende dialektale Durchschnittswerte für die 1990er Jahre: für Mainz 0,335, für Neumünster 0,288. Bei einer Analyse der Rede von Nachrichtensprechern der Tagesschau lag der Wert 1960 bei 0,04, 1990 bei 0,05. In Kostenthal liegt der durchschnittliche Dialektalitätswert bei 0,557. 
für Restdialektalität im heutigen Kostenthaler Deutsch darstellen, zu den häufigsten Sprachfehlern eines Schlesiers, der gewillt ist, Hochdeutsch zu sprechen. Zugleich wird diese Wiedergabe auch im Oberschlesisch-Deutschen konstatiert, wobei dort auf den Einfluss des schlesisch-polnischen Dialekts verwiesen wird (PELKA 2006:83). Weitgehend erhalten ist die Realisierung des ungespannten, mitteltiefen, vorderen, ungerundeten Kurzvokals $[\varepsilon]$ anstelle des standardgemäßen Reduktionsvokals [ $\mathfrak{k}$ ], etwa in abs, mutte.

Auf der morphologischen Ebene gehen auf den früheren Dialektbestand beispielsweise folgende Erscheinungen zurück: die Verwendung des bestimmten Artikels bei Personennamen, wobei dieser Usus sowohl für viele deutsche Dialekte im zusammenhängenden deutschen Sprachgebiet zutrifft (vgl. ENGEL 1999:827), als auch typisch für das Oberschlesisch-Deutsche ist (PELKA 2006:83). Im Unterschied zum Oberschlesisch-Deutschen pflegen die Kostenthaler jedoch zuerst den Nach- und dann den Vornamen zu nennen. Diese umgekehrte Reihenfolge gilt in der Literatur als landschaftlich und ist insbesondere in Süddeutschland verbreitet (FLEISCHER / HELBIG / LERCHNER 2001:664). Sehr häufig tritt die bei bestimmten Substantiven vom Standarddeutschen abweichende Pluralbildung mittels des Allomorphs $-n$ auf, etwa in zwei kretschmern, die alten kostenthalern. Gelegentlich wird davon auch bei den Familiennamen Gebrauch gemacht, und es heißt dann z. B. die himmeln. Im Oberschlesisch-Deutschen begegnen dagegen mitunter auch Fälle, in denen die Pluralkennzeichnung der Nachnamen mittels polnischer Suffixe erfolgt, weshalb dann von grammatischem Transfer gesprochen wird (PELKA 2006:149).

Im Bereich der Lexik sind nur die wenigsten Lexeme oder Wendungen exklusiv ortssprachlich wie etwa Haferfuchs als Bezeichnung für ,Heuschrecke'. Häufiger begegnen Wörter, die zwar nicht dem standarddeutschen Register zuzurechnen sind und/oder nicht einheitssprachlich sind, zugleich aber auch zum schlesischen Wortschatz gehören und im Schlesischen Wörterbuch verzeichnet sind, etwa zufleiß oder böse in der Bedeutung ,krank, schmerzend'. Abweichungen vom Standarddeutschen finden sich auch im Bereich der Syntax. Beispielsweise begegnet variabel anstelle der temporalen Subjunktion als die als landschaftlich, salopp und nicht standardsprachlich geltende Konjunktion wo: wo es meine frau hat nicht mehr ausgehalten, bloß, wo wir geflüchtet sind. Bestand hat der relativische genusindistinktive Gebrauch von was: viel sind dann gesund geworden, was nicht gesehen haben. Diese Erscheinung wird von REITER (1960:65) und PAUL (1960:402) als polnisch induziert erachtet, dagegen führt HANKE (1913:77) diese Einleitungsform der Relativsätze nicht nur als typisch für das Schlesische an, sondern auch für andere deutsche 
Felicja Księżyk

Dialekte, wie z.B. die Mundart des Vogtlandes. Zudem kommt diese Einbettungsart unter Verzicht auf Kongruenz mit dem Genus des Bezugsnomens auch in anderen deutschen Sprachinseldialekten vor, z.B. in der Südwalser Mundart von Issime. ${ }^{10}$ In Kostenthal ist diese Art der Verknüpfung nicht erst ein Phänomen der letzten Jahrzehnte, sie tritt auch in den IDS-Aufnahmen in Erscheinung und kann somit als Bewahrung des historischen Sprachbestandes gesehen werden.

Die im heutigen Kostenthalerischen vorkommenden Beispiele für Standarddivergenz stellen häufig Erscheinungen dar, die in Kostenthal zwar als Restdialektalität oder Ergebnis einer Dialektentwicklung betrachtet werden können, deren gleichzeitiges Vorkommen im Oberschlesisch-Deutschen dort allerdings auf eine polnische bzw. schlesisch-polnische Beeinflussung zurückgeführt wird. Folgende Erklärung böte sich dafür an: Der sich in vielen Teilen des deutschsprachigen Raumes vollziehende Prozess des Dialektabbaus, der darauf beruht, dass ,einige dialektale Merkmale [...] zugunsten von standardsprachlichen oder standardsprachnäheren aufgegeben [werden] [...] [, mit dem] Ergebnis [...] [, dass] ein Dialekt [entsteht], der nicht mehr lokale, sondern regionale Geltung hat" (WOLF 2002:107) ${ }^{11}$, lässt sich im Falle des Kostenthaler Deutschen nur schwer von einer Annäherung an das Oberschlesisch-Deutsche trennen. Es ist davon auszugehen, dass die Standardkonvergenz bzw. Annäherung an das Standarddeutsche zum Teil durch einen Ausgleich mit dem Oberschlesisch-Deutschen erfolgte: Bei den Trägern des (Standard-)Deutschen, mit denen die Kostenthaler sprachlich kommunizierten, handelte es sich höchstwahrscheinlich nicht allein um Reichsdeutsche, sondern in eher größerem

10 Vgl. ZÜRRER (1999:140). Dort kann allerdings das Personalpronomen das relativische Funktion übernehmen.

11 Innerhalb der mündlichen Sprachverhältnisse herrscht in den deutschsprachigen Staaten landschaftliche Unterschiedlichkeit. Dialektumbau findet im westmitteldeutschen und oberdeutschen Raum statt, wo das sprachsoziologische Prestige der Grundmundarten höher liegt. Dort werden die Basisdialekte umstrukturiert, wobei vorbildlich dafür die Schriftsprache, die Stadtdialekte oder bestimmte Regionaldialekte sind. Ergebnis dieser Umstrukturierung sind Verkehrsdialekte mit großräumiger Verbreitung, die eine Verständlichkeit im erweiterten Raum ermöglichen. In weiten Teilen des ostmitteldeutschen und niederdeutschen Raumes sowie in einigen westmitteldeutschen Teilen ist dagegen der Prozess des Dialektabbaus (anders Dialektaufgabe oder -ersatz) im Gange: Im Rheinland und im nördlichen Hessen sowie im Ostmitteldeutschen manifestiert sich das in der Ersetzung der Dialekte durch die Umgangssprache, im Niederdeutschen durch das Hochdeutsche (vgl. WIESINGER 1997:23-28). 
Ausmaß um zweisprachige Oberschlesier, die ihre Deutschkenntnisse schulmäßig erwarben. Während nämlich die Kostenthaler Dorfgemeinschaft lange Zeit ein abgeschiedenes Leben führte und ähnlich wie in anderen Sprachinseln eheliche Verbindungen lediglich innerhalb des Dorfes einging, so kam es mit der Einführung des Pflichtschulwesens (mit Deutsch als Unterrichtssprache) zu Veränderungen, die sich indirekt auf das soziale Verhalten der Kostenthaler auswirkten. Die sukzessive Zunahme der Zweisprachigkeit unter der oberschlesischen Landbevölkerung (unter den oberschlesischen Stadtbewohnern war die Zweisprachigkeit bereits früher verbreitet) ging in Kostenthal mit einer Dialektverschiebung zugunsten der Standardsprache einher, die Kostenthaler Dorfgemeinschaft wurde offener und integrierte sich in viel mehr soziale Netzwerke. Der Kontakt mit Bewohnern der umliegenden Ortschaften führte dazu, dass sprachliche Erscheinungen, die die Kostenthaler aus dem eigenen Sprachgebrauch kannten und die sie mit den zweisprachigen Oberschlesiern teilten, leichter beibehalten wurden, wohingegen exklusiv-kostenthalerische Merkmale aufgegeben wurden. Infolgedessen verfügte die Gemeinschaft über einen größeren kommunikativen Radius. Allerdings manifestiert sich der tradierte Sprachinselcharakter des Ortes bis heute darin, dass hier, wie oben angeführt, sprachliche Erscheinungen beobachtbar sind, wenn auch nicht sehr frequent, die im Oberschlesisch-Deutschen nicht begegnen.

Die Sprachinsellage Kostenthals bedeutet jedoch nicht, dass das dortige Deutsch frei von kontaktsprachigen Einflüssen wäre. Zwar beteuern die deutschstämmigen Kostenthaler immer wieder, es habe sich beim früheren Kostenthal um ein rein deutsches Dorf gehandelt, in dem nur Deutsch gesprochen worden sei. Aufgrund der im gegenwärtigen Kostenthaler Deutsch vorkommenden Sprachkontaktphänomene wäre jedoch auch diese Varietät wie das Oberschlesisch-Deutsche als Kontaktvarietät zu werten. Bei den kontaktinduzierten sprachlichen Erscheinungen handelt es sich vor allem um Nachbildungen polnischer Muster, die häufig neben standardsprachlichen Pendants auftreten und somit die Ausdrucksvielfalt erweitern. So unterliegt in der folgenden Äußerung die deutsche Präposition in nach polnischem Vorbild einer Funktionserweiterung, in der gleichen Gesprächssequenz wird aber auch die standarddeutsche Entsprechung gebraucht:

pl. w piatek (dt. , am Freitag')

am mittwoch war ich noch gewesen bei spital in coseln und in freitag wollten sie den fuß abnehmen"

Ähnliche Erscheinungen kommen auch auf lexikalischer Ebene vor, wo es zu Übernahmen der Inhaltsseite kommt, wobei die Ausdrucksseite mit eigen- 
sprachlichem Material nachgebildet wird. Es begegnen insbesondere zahlreiche Bildungen, bei denen die fremdsprachlichen Elemente Konstituente für Konstituente übersetzt werden.

pl. odbić (dt. ,kopieren')

hab ich ihn gegeben, bilder von brixen und von unserm schlosse hier, da haben sich die schulkinder abgedrückt, denn in der schule haben sie so ein dings, wie heißt das?

Derartige Spracherscheinungen beeinträchtigen zwar den Kommunikationsprozess nicht direkt, oft sind sie aber nur vor dem Hintergrund von Polnischkenntnissen verständlich. ${ }^{12}$

Neben solchen Kalkierungen kommen allerdings auch Übernahmen der Ausdrucksseite in Form lexikalischer Transfers vor. Dabei handelt es sich oft nicht um Basisvokabular, sondern um Wörter von höherer semantischer Spezifik, d.h. um Wörter, die nicht so einfach durch andere ersetzt werden können; übernommen werden auch Begriffe, die dem politisch-administrativen Bereich, der sozialistischen Arbeitsstruktur oder den modernen Lebensbereichen überhaupt zuzuordnen sind. Zumindest durch Artikelwörter werden die Lexeme morphologisch an das Deutsche als Interaktionssprache adaptiert.

pl. kierownik (dt. ,Leiter')

und in der arbeit auch, wir haben doch immer deutsch gelabert, immer deutsch und die kierowniki, die konnten auch halb deutsch, die ham verstanden alles

In dem folgenden Redeausschnitt stimmt das Genus des transferierten Substantivs weder mit dem polnischen Wort überein, noch richtet es sich nach dem deutschen Pendant. Derartige Genusschwankungen kommen im rezenten Kostenthaler Deutsch nicht nur vereinzelt vor:

$$
\begin{aligned}
& \text { pl. przystanek (mask.) (dt. ,Haltestelle }{ }^{\text {‘ }} \text { - fem.) } \\
& \text { wo jetzt das przystanek ist von den autobussen, dort war das denkmal }
\end{aligned}
$$

Hat man bei Abweichungen bei der Genuskategorie im Oberschlesisch-Deutschen häufig mit einer Nachbildung des polnischen Vorbilds zu tun (PELKA 2006:154), so scheinen die Gründe für diese Standarddivergenz im Kostenthaler Deutsch anderswo zu liegen. Es zeigt sich nämlich, dass die Artikel

12 Analoge Fälle von verdeckten Sprachkontaktfolgen beobachtet im Kansasdeutschen, das zum Teil eine englische Struktur aufweist, auch BEREND (2003). 
Die sprachliche Situation in der alten deutschen Sprachinsel Kostenthal/Gościęcin

zwar noch als Begleitwörter der Substantive erscheinen, dass sie ihre genusdeterminierende Funktion jedoch zum Teil einbüßen.

Oftmals scheint das Vorkommen von Ein-Wort-Einsprengseln im untersuchten Korpus nicht durch Nominationslücken entstanden zu sein, da die Sprecher ihre Kenntnis der deutschen Entsprechungen meist unter Beweis stellen. In manchen Beispielen ist sogar bereits der Weg zur Entdublettisierung eingeschlagen worden. So gebrauchen die Kostenthaler, obwohl ihnen das deutsche Wort Gut oder Dominium geläufig ist, häufiger das polnische Lexem majątek, während der deutsche Begriff nur bei Erklärungen oder Zusammenfassungen herangezogen wird. Von Belang kann bei der bevorzugten Verwendung des Wortes majatek seine konnotative Nebenbedeutung sein - im Unterschied zu Dominium oder Gut gehört das polnische Wort zum Bereich der sozialistischen Landwirtschaft und bezeichnet für die Sprecher unmissverständlich eine LPG. Folgt man Bellmann (1980:682), so zeugt auch die Tatsache, dass das Substantiv in hybriden Komposita vorkommt, wie im folgenden Beispiel, bereits von seiner Integration: wo der majatekmann kam. nicht? der komische. Mit einer Bedeutungsnuancierung hat man sichtlich auch im Falle des polnischen Lexems znajomy (dt. ,Bekannter') zu tun, das zusätzlich das Merkmal einer negativen Expressivität erlangt hat: no, (da hat er) blo $\beta$ immer gesoffen und gemacht mit den hm. znajomy. solche saufkollegen und da ist er alleine abgehauen.

Obwohl die Untersuchungsgruppe sich aus Angehörigen der ältesten, in der (Vor-)Kriegszeit sprachlich sozialisierten Generation zusammensetzte, die auch bei den Untersuchungen zum Oberschlesisch-Deutschen oft als der Haupt-, wenn nicht ausschließliche Träger des Deutschen gilt ${ }^{13}$, differiert der Gebrauch des Sprachrepertoires in einigen Punkten von den kommunikativen Praktiken der anderen Oberschlesier. Alle interviewten Personen geben an, sie hätten ihre Polnischkenntnisse erst in der Nachkriegszeit erworben, und tat-

13 Nach ENGERER (1996:187) hat in seiner Untersuchungsgruppe auf die Frage, „Welche Sprache haben Sie in Polen hauptsächlich benutzt?", keine in der Nachkriegszeit aufgewachsene Person Deutsch genannt. Ähnlich gilt auch in anderen Sprachinseln, etwa bei den Ungarndeutschen, die ältere Generation als Sprachbewahrer: „Dialektgebrauch hängt ähnlich wie Dialektkompetenz [...] mit der Variable ,Alter' zusammen: die ältere Generation benutzt den Dialekt noch heute in der familieninternen Kommunikation, in der jüngeren Generation dominiert hier die ungarische Standardvarietät. [...].“ (DEMINGER 2004:83) In Bezug auf das Ungarndeutsche konstatiert auch KNIPF-KomLÓsI (2003) dessen Funktionsverlust. Als dialekt-dominante Generation wertet sie einzig die älteste, die Vorkriegsgeneration, wobei auch diese sich schon abwechselnd des Ungarischen bediene. 
sächlich können die meisten von ihnen bis heute ihre Gedanken auf Deutsch präziser zum Ausdruck bringen. In dem folgenden Redeausschnitt wird auf das starke Identifikationsbewusstsein mit dem Deutschen eingegangen, das zur Folge hatte, dass viele Kostenthaler am Erlernen des Polnischen nicht interessiert waren, was wiederum Schwierigkeiten bei der sekundären Sozialisation ihrer Kinder nach sich zog:

\footnotetext{
1 H. wir haben nach der front immer deutsch gesprochen

2 T. immer nach der front wohl überall. ich konnte erscht gar nicht polnisch. kein wort.

$3 \mathrm{H}$. ich konnte doch auch nicht

4 T. ich hab erscht dann gelernt ein bissel. in der arbeit waren wir uns behauptet mit dem

$5 \quad$ deutschsprechen und fertig und da hab ich nicht gelernt, ich werd doch nicht polnisch

$6 \quad$ lernen. zu welchem teuvel! ich hab erscht dann polnisch gelernt, wo der junge anfing in die schule gehen. dann hab ich gesehen, dass ich ein btad [,Fehler'] gemacht hab. der wurde krank und kam ins krankenhaus und der konnte nicht EEIn wort, war vier jahre, der

$9 \quad$ konnte nicht ein wort polnisch (), wenn ich kam ihn nach ihn ins krankenhaus ihn

10 besuchen kurde [, verflixt noch mal!'], die haben mich ausgeschimpft und alles, der hat angst gehabt aufs

11 klo gehen, wenn das wasser gelaufen ist. alles, nicht, denn hier zu hause am topek [, Nachttopf'],

12 weil dort ins klo durfte er nicht gehen, da musste er am topek machen LUUdzie ztoty! [,Menschenskinder!']

13 da hab ich gedacht, nein, wenn der jetzt nach hause kommt, das erschte in die, in die spielschule, przedszkola [, Kindergarten']. da musste er in die przedszkola gehen, dass er halt bissel polnisch lernt und dann hab ich erscht gelernt, wo er bei den soldaten war, weil die

16 brieve gingen durch zensur, nicht? die haben doch auch kontrolliert, dann hab ich erscht gelernt. jetze kann ich auch polnisch brieve schreiben, wenn's drauf ankommt.

18 H. du kannst auch lesen nicht und alles

19 T. ach lesen sowieso, ach lesen das macht das polnische bei mir gar nichts [...] und zum
} 
Die sprachliche Situation in der alten deutschen Sprachinsel Kostenthal/Gościęcin

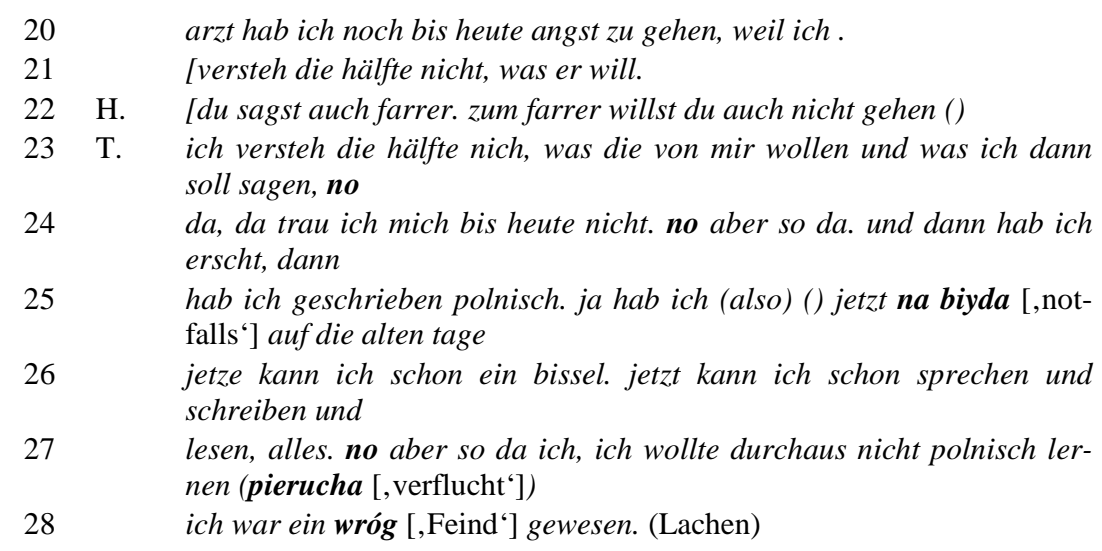

Neben den metasprachlichen Äußerungen liefert der angeführte Redeausschnitt zugleich einen Einblick in die Kommunikationspraxis der Kostenthaler, insbesondere im Hinblick auf die sprachliche Alternation, verstanden als Hyperonym zu lexikalischer Transferenz und Codeswitching. Als Codeswitching werden hier diskursfunktionale fremdsprachige Einschaltungen eingestuft; kontaktsprachige Einfügungen, die eher diskursirrelevant sind und bei denen es fraglich erscheint, ob sie von den Sprechern als einem anderen Sprachsystem zugehörig angesehen werden, gelten dagegen als Transferenz, ihnen wird vor allem referentielle Bedeutung zugesprochen (BLANKENHORN 2003:153).

Anders als Transferenz setzt Codeswitching eine vollständigere Kompetenz in der Kontaktsprache voraus (BELLMANN 1981:10). Obwohl jedoch in dem Erhebungsort heutzutage keine monolingualen Deutschsprachigen mehr leben, ist die Alternationsfrequenz bei den dort aufgenommenen Gesprächen nicht so hoch wie bei anderen zweisprachigen Gemeinschaften, etwa den Russlanddeutschen in Westsibirien oder den Sprechern des Oberschlesisch-Deutschen, die ,im Verlauf eines Gesprächs häufig zwischen den Sprachen hin und her [wechseln - F. K.]“" (BlANKENHORN 2003:11). ${ }^{14}$ Auch kommen Fälle von mehrmaligem, wechselseitigem Codeswitching, wie sie in anderen Teilen Oberschlesiens sogar in einer Gesprächssequenz oft begegnen (PELKA 2006:190), in dem untersuchten Korpus seltener vor. Da es sich bei den Kostenthalern nicht um eine Sprechergruppe handelt, in der ständig zwischen

14 Ähnliche Beobachtungen macht für Angehörige der deutschen Minderheit in Oberschlesien PELKA (2006:228). 
Felicja Księżyk

zwei Sprachen fluktuiert wird, sind auch Codewechsel, die keine kommunikative Bedeutung erkennen lassen, nicht sehr häufig. ${ }^{15}$

So lassen sich auch bei den Einschaltungen in dem obigen Redebeitrag bestimmte Zweckmäßigkeiten erkennen. Die zweimal vorkommenden Wiederholungen bzw. ,Quasi-Übersetzungen“ - in Zeile 14 spielschule - przedszkole und in Zeile 25 na biyda - auf die alten Tage - scheinen nämlich funktional eingesetzt zu sein. Während bei dem zweiten Beispiel vor allem Emphase, die Absicht, das Gesagte zu unterstreichen, eine Rolle spielen kann, so wirkt die erste Reformulierung ebenfalls verstärkend, zugleich stellt sie aber auch eine Anpassung an die offizielle Nomenklatur dar. Fälle, in denen die Sprecher bezogen auf die vorkriegszeitliche Realität deutsche Begriffe gebrauchen, in Bezug auf die Nachkriegszeit dagegen oft die offiziellen, nun gängigen Bezeichnungen anwenden, kommen häufiger vor. Sie sind als Anpassung an die gegenwärtige Welt interpretierbar und schaffen eine anschauliche Kontrastierung von Vergangenheit und Gegenwart. Die häufigste Funktion, die sich den belegten Fällen von Codeswitching zuschreiben lässt, betrifft jedoch nicht die Hervorhebung mittels Wiederholung, sondern das Zitieren. So berichtet die Sprecherin von den nachkriegszeitlichen Aussiedlungen und gibt zumindest den ersten Teil der Aussage des damaligen LPG-Leiters auf Polnisch wieder:

und wir haben halt auch unsre päcksel genommen und sind wir rausgegangen und am majatek war, wurden alle gesammelt, nicht, no und der kierownik der kam und da hat er gesagt, to mojy ludzie, mojy, mojy, hier bleiben, hier bleiben, fertig, er hatte uns nicht gelassen wegfahren [, das sind meine Leute, meine, meine ']

Das Codeshifting (d.h. der Gebrauch des Dialekts anstelle des Standarddeutschen) kommt auch beinahe ausschließlich bei der Wiedergabe fremder Rede vor. Durch das Wegsterben der ältesten Generation ist die Mundart neuerdings nämlich nicht mehr voll funktionsfähig, da des Kostenthaler Dialekts noch mächtige Personen oft keine Gesprächspartner mehr finden. Überdies dient der diskursive Einsatz von Codeswitching bei den untersuchten Gesprächen narrativen Zwecken, der Gegenüberstellung von Inhalten höherer und niedrigerer Relevanz, rhetorischer Verstärkung, der Reformulierung, der Markierung von Schlussbemerkungen, der Demonstration zweisprachiger Kompetenz u.a.m.

15 Auf diese Wechselbeziehung, und zwar, dass eine größere Häufigkeit der Codeswitchings zum Verlust von deren Qualität als Kontextualisierungshinweis führe, verweist auch FöLDES (2005:235) in seinen Untersuchungen zu ungarndeutschen Kontaktvarietäten. 
Dass die Veränderung der Sprecherkonstellation zum Codeswitching führen kann, ist in der Literatur bereits mehrfach konstatiert worden (AUER 1995:120; BLANKENHORN 2003:158-160 und RIEHL 2004:23). In Kostenthal scheint sich jedoch der bevorzugte Gebrauch des Deutschen bei manchen Sprechern darin zu offenbaren, dass sie ihre Gedanken auch dann überwiegend auf Deutsch wiedergeben, wenn sich am Gespräch eine Person beteiligt, die zwar Deutsch versteht und in bestimmten Situationen in beschränktem Maße spricht, aber ihre Redebeiträge im schlesisch-polnischen Dialekt formuliert. Einen Beleg dafür liefert der folgende Redeausschnitt (Anlass zu dem Gespräch zwischen B. und T. bildete ein Hochzeitsfoto von T.):

1 B. tacy modzi, toście byli fest modzi możno ni? [, so jung, da waren Sie wohl noch sehr jung, nicht?']

2 T. nie [,nein'], wir sin/ waren schon alt, wo wir geheiratet haben. ich war schon

3 sechsunddreißig, wo ich den jungen hatte.

$4 \quad$ B. i tyż jeszcze czas byto, ni? [, und noch gab es Zeit, nicht?']

5 T. i byto jeszcze czas [, und noch gab es Zeit'].

6 B. ja, jak se dobrze wydasz, to dobrze [, wenn du gut heiratest, dann ist

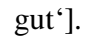

$7 \quad$ T. manchmal noch, noch zu schnell, denkt man (Lachen)

Zur Spezifik Kostenthals kann man aus linguistischer Perspektive schlussfolgern, dass das oberschlesische Deutsch keine einheitliche regionale Varietät der deutschen Gegenwartssprache darstellt, denn noch immer lässt sich das rezente Kostenthaler Deutsch qualitativ abgrenzen durch einige restdialektale Merkmale, die Zeugnis von der Konservativität dieser Varietät ablegen, und auch in der Kommunikationspraxis scheinen sich einige Unterschiede abzuzeichnen. Folgt man JOACHIMSTHALER (2002:21), so bildet jedoch das Einheitsstiftende einer Region ihre Besonderheit sowohl für Zugereiste als auch für Hineingeborene. Diese ,,schafft Identität, Loyalität, Geborgenheit und Zugehörigkeitsgefühl, [sie] sichert und bindet, fesselt und schützt". Bezüglich der Besonderheit für die Hineingeborenen kann man feststellen, dass sich die Identifikation und das Zugehörigkeitsgefühl der einheimischen Kostenthaler im Laufe der Zeit gewandelt haben. Betrachteten sie früher die Bewohner der umliegenden Ortschaften als Polnischsprecher und daher Fremde, dabei oft auf die sprachlichen Unterschiede verweisend, so gelten sie heute bei den Kostenthalern als , unsere Leute‘. Ein größeres Abgrenzungsverhältnis besteht indes gegenüber den Umsiedlern aus den ehemaligen ostpolnischen Gebieten, die sich in der Ortschaft nach dem Zweiten Weltkrieg ansiedelten. Dieses 
Felicja Księżyk

Fremdheitserleben ging vor allem auf Mentalitätsunterschiede zurück, es führte zu Verständigungsproblemen und zur kontinuierlichen Abwanderung einheimischer Kostenthaler.

Weder für das Oberschlesisch-Deutsche noch für das Kostenthaler Deutsch ist der Themenbereich für mögliche Untersuchungen ausgeschöpft. Daher sei im Folgenden auf einige Forschungsdesiderata verwiesen. Bezogen auf das Deutsch der Oberschlesier macht Pelka auf die Möglichkeit einer kontrastivkomparatistischen Untersuchung über die wechselseitigen sprachlichen Beeinflussungen aufmerksam, d.h., ob sich der Einfluss des deutsch-polnischen Sprachkontakts in beiden Richtungen manifestiert, ob sich Transferenzen also nicht nur aus dem Polnischen ins Deutsche, sondern auch aus dem Deutschen ins Schlesische oder sogar ins Standardpolnische abzeichnen. Eine Analyse, die die sprachlichen Beeinflussungen nach Altersstufen differenzieren würde, stehe ebenso noch aus. Einer eingehenden Untersuchung könnte überdies die interaktionale Kompetenz der zweisprachigen Oberschlesier im Hinblick auf die Folgen des Kontakts der verschiedenen Sprachkulturen für die Realisierung von Kulturemen ${ }^{16}$ unterzogen werden (PELKA 2006:236-239). Da sich das heutige Kostenthaler Deutsch stark an das Oberschlesisch-Deutsche angenähert hat und die Dorfgemeinschaft gegenwärtig sprachlich gemischt ist, könnte auch hier in erster Linie die gegenseitige Einflussnahme des Deutschen und Polnischen in der Redeweise der bilingualen Kostenthaler untersucht werden, etwa mit dem thematischen Schwerpunkt, inwiefern sich Unterschiede zwischen Sprachen auf lexikalische Differenzen reduzieren lassen. ${ }^{17}$ Weitere Forschungen zum kostenthalerischen Dialekt sind dagegen kaum durchführbar. Diese Varietät ist nämlich heute schon weitgehend außer Gebrauch, und im Gegensatz zu anderen Erscheinungsformen des schlesischen deutschen Dialekts findet man auch außerhalb Kostenthals keine Sprecher, die sich dieser Mundart noch in freier Rede bedienen würden. Zwar stellt TwOREK (2002:53) eindeutig fest, dass es gegenwärtig zumindest am Ort auch keinen Breslauer oder niederschlesischen deutschen Dialekt, abgesehen von idiolektalen Erscheinungen innerhalb der älteren Generation, mehr gibt. Da jedoch in manchen Regionen Deutschlands ehemalige Bewohner einiger Ortschaften Schlesiens zusammen an einem Ort leben, gibt es neuerdings einige Neuansätze zur Be-

16 Unter Kulturemen sind kulturelle Regulierungen bzw. kulturbedingte Konventionen zu verstehen, denen die sprachlich-kommunikative Verhaltensweise der Sprecher unterliegt (FöLDES 2005:271).

17 Nach MuYSKEN (1995:178) ist dies eine Frage, an deren Klärung viele Forscher interessiert sind. 
Die sprachliche Situation in der alten deutschen Sprachinsel Kostenthal/Gościęcin

schreibung des schlesischen deutschen Dialekts. Eine einleitende Untersuchung des Breslauer Deutsch, verbunden mit einer geplanten Dissertation, liefert beispielsweise MORAS (2002).

\section{Literatur}

Adelung, Johann Christoph (1809): Mithridates oder allgemeine Sprachenkunde. Teil II. Fortgesetzt und bearbeitet von Johann Severin Vater. Hildesheim/New York 1970 (=Nachdruck der Ausgabe Berlin 1809), 216-218; 668-670.

[ANONYM] (2002) [=Jürgen Joachimsthaler, Aleksandra Bochenek, Jarosław Bogacki, Ulrike Fügl, Gabriela Jelitto, Grzegorz Jureczko, Maria K. Lasatowicz, Sebastian Mrożek, Daniela Pelka, Michael Rohrwasser, Andrea Rudolph, Izabela Surynt, Magdalena Sutarzewicz, Wiesław Śliwak, Monika Witt, Marek Zybura und Janusz Zydroń] (eds.): Regionalität als Kategorie der Sprach- und Literaturwissenschaft. Hrsg. vom Instytut Filologii Germańskiej der Uniwersytet Opolski. Frankfurt (M.)/Berlin/Bern u. a.

AUER, PETER (1995): The pragmatics of code-switching: a sequential approach. In: MUYSKEN/ MiLROY, 115-135.

BELlmANN, GÜNTER (1971): Slavoteutonica. Lexikalische Untersuchungen zum slawisch-deutschen Sprachkontakt im Ostmitteldeutschen. Berlin/New York.

- (21980): Slawische Sprachen und deutsche Gesamtsprache. In: Althaus, Hans Peter / Henne, Helmut / Wiegand, Herbert ERnst (eds.): Lexikon der germanistischen Linguistik. Studienausgabe IV. Tübingen, 680-685.

- (1981): Sprachkontakt und Semantik. In: MeID, WolfGang / Heller, Karin (eds.): Sprachkontakt als Ursache von Veränderungen der Sprach- und Bewußtseinstruktur. Eine Sammlung von Studien zur sprachlichen Interferenz. Innsbruck (=Innsbrucker Beiträge zur Sprachwissenschaft 34), 9-18.

BEREND, NINA (2003): Zur Vergleichbarkeit von Sprachkontakten: Erfahrungen aus wolgadeutschen Sprachinseln in den USA und Russland. In: KeEL/ MATTHEIER, 239-268.

Blankenhorn, RenAte (2003): Pragmatische Spezifika der Kommunikation von Russlanddeutschen in Sibirien. Entlehnung von Diskursmarkern und Modifikatoren sowie Code-switching. Frankfurt (M.) (=Berliner Slawistische Arbeiten 20).

DEMINGER, SZILVIA (2004): Spracherhalt und Sprachverlust in einer Sprachinselsituation. Sprache und Identität bei der deutschen Minderheit in Ungarn. Frankfurt (M.) (=Variolingua. Nonstandard-Standard-Substandard 21).

EBERTH, KARL (1846): Ueber die gewöhnlichsten Sprachfehler der Schlesier. In: Schlesische Provinzialblätter 123:217-226.

Engel, Ulrich et al. (1999): Deutsch-polnische kontrastive Grammatik. 2 Bde. Heidelberg/Warszawa.

ENGERER, VOLKMAR (1996): Sprachwechsel in Oberschlesien. Eine Befragung von Aussiedlern. Berlin. 
Fleischer, Wolfgang / Helbig, Gerhard / Lerchner, GotThard (eds.) (2001): Kleine Enzyklopädie Deutsche Sprache. Frankfurt (M.) u. a.

FÖLDES, CSABA (2002): Aspekte der Regionalität im System der diatopischen Varietäten der deutschen Sprache. In: [ANONYM], 77-95.

- (2005): Kontaktdeutsch. Zur Theorie eines Varietätentyps unter transkulturellen Bedingungen von Mehrsprachigkeit. Tübingen.

FRIEMEL, WILHELM (1937): Die mundartlichen Beziehungen von Kostenthal als Quelle siedlungsgeschichtlicher Zusammenhänge. In: Der Oberschlesier 19/10:592-595.

- (1937a): Die Mundart von Oberglogau. In: Schlesische Geschichtsblätter 3:104-108.

- (1938): Kostenthal im oberschlesischen Sprachraum. In: Der Oberschlesier 20:107-113.

GRAEBISCH, Friedrich (1929): Proben der deutschen Mundarten Oberschlesiens. In: Der Oberschlesier 11:120-128.

HAŁUb, MAReK (ed.) (2002): Silesia Philologica. I Kongres Germanistyki Wroctawskiej. [1. Kongress der Breslauer Germanistik]. Wrocław (=Acta Universitatis Wratislaviensis 2386).

HANKe, LothaR (1913): Die Wortstellung im Schlesischen. Breslau (=Wort und Brauch. Volkskundliche Arbeiten namens der Schlesischen Gesellschaft für Volkskunde 11).

HERRGEN, JOACHIM / SCHMIDT, JÜRGEN E. (1985): Systemkontrast und Hörerurteil. Zwei Dialektalitätsbegriffe und die ihnen entsprechenden Meßverfahren. In: Zeitschrift für Dialektologie und Linguistik 52:20-42.

Herrgen, Joachim / Lameli, Alfred et al. (2001): Dialektalität als phonetische Distanz. Ein Verfahren zur Messung standarddivergenter Sprechformen, 1-11: www.unimarburg.de/dsa/papers/Dialektalitaetsmessung.pdf (06.05.2005).

JoACHIMSTHALER, JÜRgen (2002): Die Literarisierung einer Region und die Regionalisierung ihrer Literatur. In: [ANONYM], 17-49.

Keel, William D. / Mattheier, Klaus J. (eds.) (2003): German Language Varieties Worldwide: Internal and external Perspectives. Deutsche Sprachinseln weltweit: Interne und externe Perspektiven. Frankfurt (M.) u. a.

KNEIP, MATTHIAS (2000): Die deutsche Sprache in Oberschlesien. Untersuchungen zur politischen Rolle der deutschen Sprache als Minderheitensprache in den Jahren 19211998. Dortmund.

KNIPF-Komlósi, ELISABETH (2003): Sprachwahl und kommunikative Handlungsformen der deutschen Minderheit in Ungarn. In: KeEL / MATTHEIER, 269-281.

KuHN, WALTER (1934): Deutsche Sprachinselforschung. Geschichte, Aufgaben, Verfahren. Plauen im Vogtland (=Ostdeutsche Forschungen 2).

LAMELI, AlFRed (2003): Standard und Regionalsprache - Konstanz und Wandel, 1-17: http://www.uni-marburg.de/dsa/papers/iggd_netz.pdf (13.05.2005).

MAK, WILHELM (1929): Die Kostenthaler Mundart ist gefährdet. In: Der Oberschlesier 11:140-141. 
Die sprachliche Situation in der alten deutschen Sprachinsel Kostenthal/Gościęcin

MATTHEIER, KLAUS J. (2003): Sprachinseltod: Überlegungen zur Entwicklungsdynamik von Sprachinseln. In: KEEL / MATTHEIER, 13-31.

Moras, AgnieszKa (2002): Niemczyzna wrocławska - utracony język. [Das Breslauer Deutsch - eine verlorene Sprache]. In: HaŁub, 60-66.

MORCINIEC, NORBERT (1999): Zum deutsch-polnischen Sprachkontakt in Schlesien. In: Lasatowicz, Maria Katarzyna / Joachimsthaler, JÜrgen (eds.): Assimilation Abgrenzung - Austausch. Interkulturalität in Sprache und Literatur. Frankfurt (M.) (=Oppelner Beiträge zur Germanistik 1), 287-303.

MUYSKEN, PIETER (1995): Code-switching and grammatical theory. In: MUYSKEN / MiLROY, 177-198.

MuYSKen, PIETER / MiLRoy, LESLEy (eds.) (1995): One speaker, two languages. Crossdisciplinary perspectives on code-switching. Cambridge.

Olesch, ReINHOLD (1970/1992): Slavistische Anmerkungen zum Schlesischen Wörterbuch. In: OLESCH, 192-205.

- (1987/1992): Interferenz und Integration im deutsch-polnischen Kontaktraum Oberschlesien. In: OLESCH, 252-264.

Olesch, ReInHOLD (1992): Gesammelte Aufsätze. Hrsg. von Angelika Lauhus. Köln/ Weimar/Wien (=Slavistische Forschungen 59).

PAul, HeRMANN ( $\left.{ }^{6} 1960\right)$ : Prinzipien der Sprachgeschichte. Tübingen.

PelKa, Daniela (2006): Der deutsch-polnische Sprachkontakt in Oberschlesien am Beispiel der Gegend von Oberglogau. Berlin (=SILESIA. Schlesien im europäischen Bezugsfeld. Quellen und Forschungen 2).

REITER, NORBERT (1960): Die polnisch-deutschen Sprachbeziehungen in Oberschlesien. Wiesbaden.

RIEHL, ClaUdia MARIa (2004): Sprachkontaktforschung. Eine Einführung. Tübingen.

ROHFLEISCH, IRENE (2001): Sprachsituation und Sprachverhalten in Teilen des heutigen Oberschlesiens. Heidelberg.

SCHERHOLZ, JosEF (1937): Unser Kostenthalheft. In: Der Oberschlesier 19/10:543-545. SCHWARZ, ERnst (1935): Sudetendeutsche Sprachräume. München.

TRABANT, JÜRgEN: Mithridates in Berlin: http://www.berliner-klassik.de/projekte/ publikationen/online/trabant (30.07.2007).

TwOreK, Artur (2002): (Dolno)ślaski niemiecki? (Dolno)ślaski polski? O wspótczesnej wymowie Dolnoślazaków. [(Nieder)schlesisches Deutsch? (Nieder)schlesisches Polnisch? Über die gegenwärtige Aussprache der Niederschlesier]. In: HAŁUB, 50-59.

Weinelt, HERBERT (1938): Sprache und Siedlung der oberschlesischen Sprachinsel Kostenthal. In: Brackmann, Albert / Hassinger, Hugo / Metz, Friedrich (eds.): Deutsches Archiv für Landes- und Volksforschung. Leipzig, 386-403.

- (1939): Zur Herkunftsbestimmung alter deutscher Volkinseln im ostmitteldeutschen Vorland auf Grund der Mundart. In: Zeitschrift für Mundartforschung 15:25-39. 
Felicja Księżyk

Wiesinger, Peter (1997): Sprachliche Varietäten - Gestern und Heute. In: STICKeL, GERHARD (ed.): Varietäten des Deutschen. Regional- und Umgangssprachen. Berlin/New York (=IDS Jahrbuch 1996), 9-45.

WikToRowicz, Józef (2001): Die Historiographie der deutsch-polnischen Sprachbeziehungen vor dem Hintergrund der politischen Geschichte der beiden Länder. In: GruCZA, FrancisZeK (ed.): Tausend Jahre polnisch-deutsche Beziehungen. Sprache Literatur - Kultur - Politik. Warszawa, 265-275.

WOLF, NORBERT RICHARD (2002): Regionen entstehen, indem die Leute miteinander reden. Oder: Regionalisierungsprozesse in rezenten Dialekten. In: [ANONYM], 97-110.

ZÜRRER, PETER (1999): Sprachinseldialekte: Walserdeutsch im Aostatal (Italien). Aarau/Frankfurt (M.)/Salzburg (=Reihe SPL 23): http://www.ids-mannheim.de/DSAv (19.07.2007). 